\title{
A Comparison of Hyperextension versus Neutral Positions for Vertebroplasty to Treat Neurologically Intact Kümmell Disease
}

\author{
Yan Ding ${ }^{1}$, Shengjie Dong ${ }^{1}$, Jingjie Wang ${ }^{1}$, Jinpeng Cuil ${ }^{2}$, Zhilin Cao ${ }^{2}$ and Shiqiao Lv $^{1}$ \\ ${ }^{1}$ Department of Orthopaedics, Yantaishan Hospital, Yantai, China \\ ${ }^{2}$ Department of Clinical Laboratory, Yantaishan Hospital, Yantai, China
}

\begin{abstract}
Objective: To compare the efficacy between hyperextension position (HPVP) and neutral position for vertebroplasty (NPVP) in treating Kümmell disease.

Study Design: A Comparative descriptive study.

Place and Duration of Study: Department of Orthopaedics, Yantaishan Hospital, China, from December 2017 to July 2018.

Methodology: This study retrospectively analysed demographic features, operative information, radiologic data, and complications of 58 consecutive patients with single-level Kummel disease (KD) who underwent NPVP $(n=27)$ or HPVP $(n=31)$. All patients were observed preoperatively and at 2 days (POD 2) and one year postoperatively for cement leakage, Cobb's angle, antenior body height ratio, pain and disability.

Results: The cement leakage rate was significantly lower in the HPVP group $(p<0.01)$. The visual analogue scale (VAS) scores for pain, and Oswestry disability indices (ODIs) were lower in the HPVP group at one year postoperatively (both $p<0.05$ ). For the HPVP group, Cobb's angle was significantly smaller, and the anterior body height ratio (AR) was larger at POD $2(p<0.05)$ and one year $(p<0.05)$, postoperatively.

Conclusion: This study demonstrated that HPVP could achieve a lower cement leakage rate with similar operative time, lower VAS and ODI scores, as well as better kyphosis restoration and AR recovery at the 1-year follow-up than NPVP. HPVP is thus superior to NPVP in treating KD.
\end{abstract}

Key Words: Kyphosis, Cobb's angle, Cement leakage, Intervertebral stability, Surgical outcome, Kümmell disease, Vertebroplasty, Hyperextension position.

How to cite this article: Ding Y, Dong S, Wang J, Cui J, Cao Z, Lv S. A Comparison of Hyperextension versus Neutral Positions for Vertebroplasty to Treat Neurologically Intact Kümmell Disease. J Coll Physicians Surg Pak 2020; 30(11):1155-1160.

\section{INTRODUCTION}

Kümmell disease $(K D)$ is defined as a delayed, traumatic vertebralcollapse. ${ }^{1}$ Themain pathomorphological changes are vertebral collapse and gradual kyphosis progression occurring after a period of asymptomatic minor spinal trauma. ${ }^{2}$ The treatment targets are to relieve back pain, attain intervertebral stability to prevent vertebral collapse, and avoid spinal kyphosis progression and neurological deficits. ${ }^{3}$ Given that patients with KD are generally old and may have various comorbidities, minimally invasive surgeries, including percutaneous vertebroplasty (VP) or kyphoplasty (KP), ${ }^{4}$ are widely accepted as the first-line treatmentfor KD without neurological symptoms. ${ }^{2,4}$

Correspondence to: Dr. Shiqiao Lv, Department of Orthopaedics, Yantaishan Hospital, No. 91 Jiefang Road, Yantai 264000, China

E-mail:68402128@qq.com

Received: July 20, 2020; Revised: September 28, 2020;

Accepted: November 02, 2020

DOI: https://doi.org/10.29271/jcpsp.2020.11.1155
One of the main disadvantages of both KP and VP is the difficulty in achieving satisfactory restoration. Therefore, some researchers performed $\mathrm{KP}^{2,3,5-7}$ or $\mathrm{VP}^{8}$ in the hyperextension position (HPVP) to achieve better restoration and reported satisfactory results. A previous study compared the parameters of HPVP before and after the operation and reported that this procedure was effective. ${ }^{8}$ However, to the authors' knowledge, no study has compared the surgical effects, radiographic parameters, and complications between HPVP and VP in the neutral position (NPVP). Thus, it is inconclusive that HPVP is indeed superior to NPVP. Therefore, this study aimed to compare the efficacy between HPVPand NPVP in treating KD.

\section{METHODOLOGY}

This study retrospectively reviewed 58 consecutive patients diagnosed with single-level KD and underwent VPat Department of Orthopaedics, Yantaishan Hospital, China from December 2017 to July 2018. As most studies have agreed and reported that the main pathologic mechanisms of KD are ischaemic vertebral necrosis and intravertebral pseudarthrosis after osteoporotic fractures, ${ }^{9}$ computed tomography (CT) and magnetic resonance imaging (MRI) were both performed to clarify the existence of the 
intravertebral vacuum phenomenon and osteosclerotic zone in this study. The patients whose CT and MRI images showed the abovementioned radiographic findings were included in the study. Patients were excluded, if they had an infection, primary and metastatic spinal tumours, multiple myeloma, more than one vertebral level affected, and neurological symptoms present.

All patients were observed and assessed preoperatively, on postoperative day: 2 (POD 2; after they stepped out of bed) and one year postoperatively. The first 27 patients underwent NPVP. Thereafter, the authors learned that HPVP might be more beneficial to the patients; thus, the remaining 31 patients underwent HPVP.

This study was conducted in accordance with guidelines stipulated in the World Medical Association Declaration of Helsinkiand approved by the Ethics Committee of Yantaishan Hospital (approval No. 2020002). Written consents for the publication of the images included in this study were obtained from the patients and their relatives.

Data on demographic features (age, gender, body mass index (BMI), and duration from trauma to surgery), operative information (level of affected vertebrae, operative time, and injected cement volume), radiologic data (bone mineral density (BMD), and preoperative and postoperative $X$-ray images), and complications (cement leakage and re-fracture) were collected and analysed for the two groups. Back pain was assessed using the visual analogue scale (VAS), and the impact on patients' daily life was evaluated using the Oswestry Disability Index (ODI). To eliminate individual differences, the authors compared the ratios of the anterior vertebral body (AR, anterior height of the vertebral body/posterior height of the vertebral body on X-ray image) and middle vertebral body (MR, middle height of the vertebral body/posterior height of the vertebral body on X-ray image), instead of the absolute heights of the anterior and middle vertebral bodies. These two ratios and the vertebral Cobb's angle, which is the angle between the horizontal line of the upper vertebral body's upper endplate and the lower vertebral body's lower endplate, taking the affected vertebrae as the centre, were measured using lateral $\mathrm{X}$-ray images, and these were the main radiographic parameters compared. Preoperative CT in the hyperextension position was also performed to determine the degree of restoration during the operation. Cement leakage was obtained by observing the postoperative X-ray images; any cement noticed outside the vertebral body was considered positive for cement leakage, including minimal leakage extending from the main cement mass, leakage into the intervertebral space, and leakage in the vessel around the vertebrae. Re-fracture was defined as the re-appearance of pain symptoms in the 1-year-follow-up and confirmed by MRI.

Preoperatively, both groups were trained to lie in the prone position twice daily and maintain the position for approximately 30 min in order to tolerate surgery. ${ }^{3,4}$ In HPVP, the patient was placed in a prone position with multiple pillows under the chest and ilium until the abdomen was fully suspended with one solid paddle under the thigh to maintain the whole-body hyperextension posi- tion. After sterilisation, local anaesthesia ( $0.5 \%$ lidocaine) was induced by multipoint injections. Then, the operator gently pressed the patient's back until the anterior height of the vertebral body was near the images shown on the pre-operation CT in the hyperextension position, and the assistant pushed the patient's back to maintain the hyperextension position. Subsequently, VP was performed through a unilateral transverse process-pedicle approach. ${ }^{10-12}$ The injection process was monitored continuously under G-arm and stopped immediately if high resistance was encountered, or polymethylmethacrylate (PMMA) spread was observed near the posterior wall of the vertebral body. Postoperatively, the patients were kept on bed rest for approximately 6 hours and then stepped out of bed without brace support. Atypical case is presented in Figure 1.

For NPVP, the patient was placed in a prone position, and VP was also performed through a unilateral transverse process-pedicle approach without diaplasis.

The quantitative variables were expressed as mean \pm standard deviation and the qualitative variables as frequencies and percentages. Paired t-tests were performed to compare the VAS, ODI, Cobb's angle, AR, and MR in the same group. Chi-squared/Fisher Exact tests were performed to compare the sex distribution, the affected level, cement leakage rate, and re-fracture rate at the final follow-up between the two groups. Independent samplet-tests were used to compare patients' age, BMI, BMD, KD duration, operative time, and injected bone cement volume between the two groups. The VAS, ODI, Cobb's angle, AR, and MR at three timepoints (preoperatively, POD2, and 1-year follow-up) between the groups were also analysed using an independent sample t-test. SPSS version 25.0 (IBM Corp., Armonk, NY, USA) was used for statistical analysis. The significance level of the $p$ value was set at 0.05 .

Table I: Patients' characteristics and operative parameters.

\begin{tabular}{|c|c|c|c|}
\hline Variables & NPVP & HPVP & $p$-value \\
\hline Number & 27 & 31 & \\
\hline Age (years) & $69.89 \pm 7.89$ & $69.65 \pm 7.17$ & 0.902 \\
\hline \multicolumn{3}{|l|}{ Sex } & \multirow{3}{*}{0.507} \\
\hline Male & $5(18.52 \%)$ & $8(25.81 \%)$ & \\
\hline Female & $22(81.48 \%)$ & $23(74.19 \%)$ & \\
\hline BMI $\left(\mathrm{kg} / \mathrm{m}^{2}\right)$ & $24.48 \pm 2.77$ & $23.73 \pm 2.68$ & 0.301 \\
\hline BMD & $-2.81 \pm 0.87$ & $-2.31 \pm 1.17$ & 0.075 \\
\hline $\begin{array}{l}\text { Duration } \\
\text { (months) }\end{array}$ & $5.12 \pm 0.85$ & $5.30 \pm 1.09$ & 0.489 \\
\hline \multicolumn{3}{|l|}{ Affected level } & \multirow{7}{*}{0.599} \\
\hline T10 & $0(0.00 \%)$ & $1(3.23 \%)$ & \\
\hline T11 & $6(22.22 \%)$ & $6(19.35 \%)$ & \\
\hline T12 & $8(29.63 \%)$ & $6(19.35 \%)$ & \\
\hline L1 & $7(25.93 \%)$ & $13(41.94 \%)$ & \\
\hline L2 & $5(18.52 \%)$ & $3(9.68 \%)$ & \\
\hline L4 & $1(3.70 \%)$ & $2(6.45 \%)$ & \\
\hline $\begin{array}{l}\text { Operative time } \\
\text { (min) }\end{array}$ & $20.70 \pm 1.79$ & $21.10 \pm 2.02$ & 0.440 \\
\hline $\begin{array}{l}\text { Cement } \\
\text { volume }(\mathrm{mL})\end{array}$ & $6.63 \pm 0.67$ & $7.15 \pm 0.44$ & $0.001^{\mathrm{a}}$ \\
\hline $\begin{array}{l}\text { Cement } \\
\text { leakage }\end{array}$ & $22(81.48 \%)$ & $13(41.94 \%)$ & $0.002^{\mathrm{a}}$ \\
\hline Re-fracture & $2(7.41 \%)$ & $0(0.00 \%)$ & 0.212 \\
\hline \multicolumn{4}{|c|}{$\begin{array}{l}\text { BMI: Body mass index, BMD: Bone mineral density, HPVP: Vertebroplasty } \\
\text { in the hyperextension position, NPVP: Vertebroplasty in the neutral } \\
\text { position. }{ }^{a} p<0.01 \text {. }\end{array}$} \\
\hline
\end{tabular}


Table II. Statistical analysis at three different time points between the two groups and within the NPVP or HPVP group with $p$-values of related parameters.

\begin{tabular}{|c|c|c|c|}
\hline Variables & NPVP & HPVP & P-value \\
\hline Number & 27 & 31 & \\
\hline Pre-op VAS & $5.89 \pm 2.33$ & $6.00 \pm 1.63$ & 0.836 \\
\hline Pre-op ODI (\%) & $60.99 \pm 21.43$ & $65.56 \pm 16.54$ & 0.363 \\
\hline Pre-op Cobb & $15.76 \pm 5.04$ & $16.67 \pm 4.85$ & 0.485 \\
\hline Pre-op AR (\%) & $62.47 \pm 11.62$ & $58.35 \pm 12.77$ & 0.207 \\
\hline Pre-op MR (\%) & $54.01 \pm 10.69$ & $47.63 \pm 14.27$ & 0.057 \\
\hline POD 2 VAS & $1.78 \pm 0.80$ & $1.45 \pm 0.68$ & 0.098 \\
\hline POD 2 ODI (\%) & $17.19 \pm 8.49$ & $12.36 \pm 10.56$ & 0.063 \\
\hline POD 2 Cobb & $14.19 \pm 4.66$ & $10.47 \pm 4.47$ & $0.003^{b}$ \\
\hline POD 2 AR (\%) & $68.47 \pm 10.51$ & $74.37 \pm 10.14$ & $0.034^{\mathrm{a}}$ \\
\hline POD 2 MR (\%) & $59.86 \pm 8.07$ & $63.30 \pm 10.89$ & 0.182 \\
\hline Last follow-up VAS & $1.59 \pm 0.64$ & $1.23 \pm 0.72$ & $0.045^{a}$ \\
\hline Last follow-up ODI (\%) & $16.99 \pm 7.32$ & $11.03 \pm 8.76$ & $0.007^{b}$ \\
\hline Last follow-up Cobb & $14.41 \pm 4.68$ & $10.63 \pm 4.55$ & $0.003^{b}$ \\
\hline Last follow-up AR (\%) & $67.53 \pm 10.73$ & $73.24 \pm 10.58$ & $0.046^{\mathrm{a}}$ \\
\hline Last follow-up MR (\%) & $59.67 \pm 7.97$ & $63.12 \pm 10.84$ & 0.179 \\
\hline \multirow{15}{*}{ NPVP } & \multirow{3}{*}{ VAS } & Pre-op vs POD 2 & $<0.001^{b}$ \\
\hline & & Pre-op vs 1-year follow-up & $<0.001^{\mathrm{b}}$ \\
\hline & & POD 2 vs 1-year follow-up & $0.022^{a}$ \\
\hline & \multirow{3}{*}{ ODI } & Pre-op vs POD 2 & $<0.001^{\mathrm{b}}$ \\
\hline & & Pre-op vs 1-year follow-up & $<0.001^{\mathrm{b}}$ \\
\hline & & POD 2 vs 1-year follow-up & 0.867 \\
\hline & \multirow{3}{*}{ Cobb } & Pre-op vs POD 2 & $0.001^{\mathrm{b}}$ \\
\hline & & Pre-op vs 1-year follow-up & $0.002^{b}$ \\
\hline & & POD 2 vs 1-year follow-up & $<0.001^{b}$ \\
\hline & \multirow{3}{*}{$A R$} & Pre-op vs POD 2 & $<0.001^{\mathrm{b}}$ \\
\hline & & Pre-op vs 1-year follow-up & $<0.001^{b}$ \\
\hline & & POD 2 vs 1-year follow-up & $<0.001^{\mathrm{b}}$ \\
\hline & \multirow{3}{*}{ MR } & Pre-op vs POD 2 & $<0.001^{b}$ \\
\hline & & Pre-op vs 1-year follow-up & $<0.001^{\mathrm{b}}$ \\
\hline & & POD 2 vs 1-year follow-up & $<0.001^{b}$ \\
\hline \multirow{15}{*}{ HPVP } & \multirow{3}{*}{ VAS } & Pre-op vs POD 2 & $<0.001^{b}$ \\
\hline & & Pre-op vs 1-year follow-up & $<0.001^{b}$ \\
\hline & & POD 2 vs 1-year follow-up & $0.006^{b}$ \\
\hline & \multirow{3}{*}{ ODI } & Pre-op vs POD 2 & $<0.001^{\mathrm{b}}$ \\
\hline & & Pre-op vs 1-year follow-up & $<0.001^{\mathrm{b}}$ \\
\hline & & POD 2 vs 1-year follow-up & $0.028^{a}$ \\
\hline & \multirow{3}{*}{ Cobb } & Pre-op vs POD 2 & $<0.001^{b}$ \\
\hline & & Pre-op vs 1-year follow-up & $<0.001^{b}$ \\
\hline & & POD 2 vs 1-year follow-up & $<0.001^{\mathrm{b}}$ \\
\hline & \multirow{3}{*}{$A R$} & Pre-op vs POD 2 & $<0.001^{b}$ \\
\hline & & Pre-op vs 1-year follow-up & $<0.001^{\mathrm{b}}$ \\
\hline & & POD 2 vs 1-year follow-up & $<0.001^{\mathrm{b}}$ \\
\hline & \multirow{3}{*}{ MR } & Pre-op vs POD 2 & $<0.001^{b}$ \\
\hline & & Pre-op vs 1-year follow-up & $<0.001^{\mathrm{b}}$ \\
\hline & & POD 2 vs 1-year follow-up & 0.059 \\
\hline
\end{tabular}

\section{RESULTS}

The correlation data conformed to a normal distribution, according to the results of the Levene's test. The demographic and operative data of the groups and the comparison values between the two groups are summarised in Table I. The difference in mean age, gender distribution, BMI, BMD, KD duration, affected vertebral levels, operative time, and re-fracture rate between the two groups at the one-year follow-up was not significant. However, the injected bone cement volume was significantly higher in the HPVP group than in the NPVP group (7.15 \pm 0.44 vs. $6.63 \pm 0.67 \mathrm{~mL}, p=0.001)$. The cement leakage rate was significantly lower in the HPVP group than in the NPVP group (41.94\% [13/31] vs. 81.48\% [22/27]; $p=0.002$ ). No neurological deficit, pulmonary embolism, and cement loosening were observed in both groups. None of the patients in the HPVP group had re-fractures, while two non-adjacent vertebral fractures were observed in the NPVP group at the 1-year-follow-up.

The exact value and comparison of VAS scores, ODI, Cobb's angle, AR, and MR in the same group at three time points, and between the two groups are presented in Table II. 


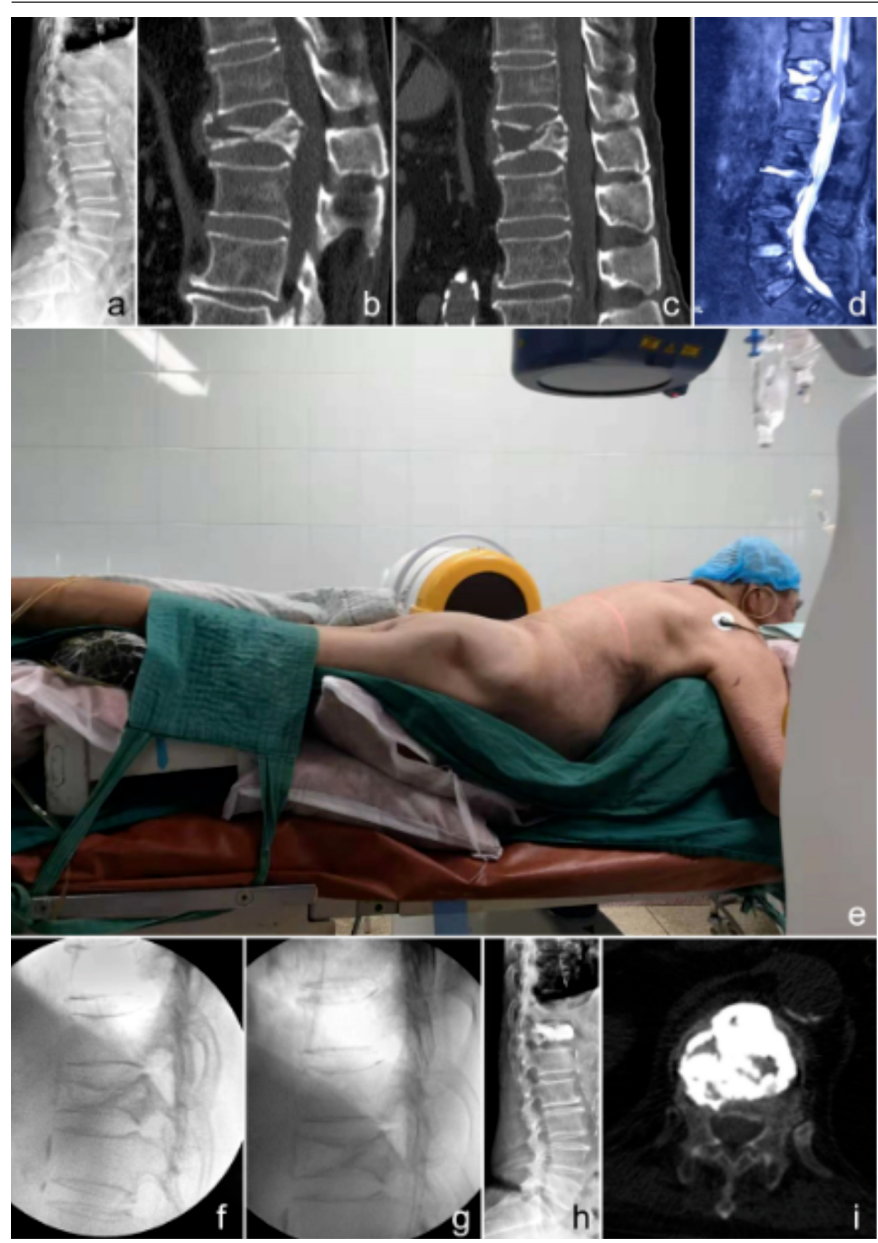

Figure 1: A representative case. A 76-year-old male patient complained of back pain for more than half a year, which particularly occurred upon changing position. Physical examination showed an intact neurological function. (a) Preoperative X-ray in the lateral position. (b) Preoperative computed tomography (CT) in the neutral position showing a broken bone block that herniated into the spinal canal. (c) CT in the hyperextension position: the herniated bone block is more or less restored, and the spinal canal occupation is decreased. This image served as the standard to guide the restoration during operation. (d) Preoperative T2-weighted magnetic resonance (MR) image showing a liquidity signal. (e) Hyperextension position during the operation with G-arm positioned. (f) Lateral X-ray during the operation before diaplasis. (g) In a lateral X-ray during the operation after diaplasis, restoration of the anterior vertebral body height can be observed compared with image e. (h) Lateral Xray after the operation. (i) Horizontal view of a CT scan after the operation.

As also seen in that Table, no significant difference was found preoperatively among the parameters mentioned above $(p>0.05)$.

The VAS scores showed a decreasing trend at the three time points, and the values were significantly different $(p<0.05)$ in both groups. No significant difference in VAS scores between the two groups was observed on POD 2; however, VAS scores at the 1-year follow-up were significantly lower in the HPVP group than in the NPVP group $(p<0.05)$.

As for the ODI, a decreasing trend was observed at the three time points in the HPVP group, showing significant differences $(p<0.05)$. The decreasing preoperative values in both groups were significantly different than those obtained on POD 2 and at the 1-year follow-up $(p<0.01)$. However, no significant difference was found when the values on POD 2 were compared with those at the 1-year follow-up in the NPVP group $(p>0.05)$. No significant differences in ODI on POD 2 were noted between the two groups; however, at the 1-year follow-up, the ODI was significantly lower in the HPVP group $(p<0.05)$.

In both groups, Cobb's angle decreased on POD $2(p<0.01)$, but it slightly increased at the 1 -year follow-up $(p<0.01)$. In both groups, a significant difference in Cobb's angle was found both on POD 2 and at the 1-year follow-up $(p<0.01)$. The Cobb's angle was significantly smaller in the HPVP group than in the NPVP group.

In both groups, a favourable AR was obtained on POD 2, compared with that obtained preoperatively $(p<0.01)$, which was partly maintained until the 1-year follow-up. However, height loss was significantly different between the two groups $(p<0.01)$. The AR was significantly larger in the HPVP group than in the NPVP group, both on POD 2 and at the 1year follow-up $(p<0.05)$.

In both groups, the MR showed recovery on POD 2, compared with that obtained preoperatively $(p<0.01)$, partly maintained until the 1-year follow-up. However, there were significant differences in height loss among the three time points in the NPVP group $(p<0.01)$, but not in the HPVP group $(p>0.05)$. In the between-group comparisons, no significant difference in MR values was found both on POD 2 and at the 1-year follow-up ( $p>0.05$ ).

\section{DISCUSSION}

In the analysis of VAS scores, ODI, Cobb's angle, AR, and MR before and after the operation, both NPVP and HPVP were shown to be effective treatments for KD. For the VAS and ODI scores, the effects of NPVP and HPVP were comparable between the two groups on POD 2; nevertheless, the HPVP group had lower VAS and ODI values than the NPVP group at the one-year follow-up. As for Cobb's angle, HPVP could attain better kyphosis restoration than NPVP, which was maintained until the 1-year follow-up. Moreover, HPVP patients showed better AR recovery than NPVP patients, which was maintained until the one-year follow-up; the main complication and cement leakage rates were also lower in the HPVP group. Therefore, HPVP is a better treatment choice for KD than NPVP.

The thoracolumbar junction is the most commonly affected segment in KD. ${ }^{13}$ This junctional area is neutral under normal conditions, but kyphosis occurs in KD cases. Some authors found that vertebral deformities can cause substantial back pain. ${ }^{14}$ Previous studies have demonstrated that segmental kyphosis shortened the anterior column and increased the load on the adjacent vertebral bodies, which would increase the risk of adjacent segment fractures. ${ }^{15,16}$ Thus, maximum 
kyphosis correction should be a target of KD treatment. In the present study, both the NPVP and HPVP groups could achieve kyphosis correction to some extent, and the VAS and ODI values declined equally on POD 2, which was possibly because the bone cement acted as a glue to attain intervertebral stability in both groups. ${ }^{3}$ However, the longterm outcome showing that VAS and ODI values were lower in the HPVP group than in the NPVP group may be because the kyphosis correction in the HPVP group was better than that of the NPVP group. Similar results were also reported in previous studies. ${ }^{4,6,7}$

For the AR and MR, the results of the present study showed that AR recovery was better in the HPVP group than in the NPVP group, which may be related to kyphosis recovery. When the patient was placed in the hyperextension position, the anterior vertebral body could be restored under the stretch stress of the anterior longitudinal ligament. ${ }^{17}$ However, the mechanism does not exist in the middle of the vertebral body. This may be the reason why AR recovery was better in the HPVP group, but MR recovery was comparable between the two groups.

Cement leakage is one of the main complications of VP. Park et al. reported that the cement leakage rate was $26.3 \%$ with a mean bone cement injection of $3.9 \mathrm{~mL} .{ }^{8}$ Zhang et al. ${ }^{3}$ reported that in HPVP with $3.23 \pm 0.94 \mathrm{~mL}$ of bone cement, the cement leakage rate was $36.4 \%(8 / 22)$, whereas in hyperextension KP with $3.04 \pm 1.00 \mathrm{~mL}$ of bone cement, the cement leakage rate was $23.1 \%$ (3/13). The amount of injected bone cement and the leakage rate in their study were lower than those of the present study. The cement volume of this study was $6.63 \pm 0.67 \mathrm{~mL}$ in the NPVP group and $7.15 \pm 0.44 \mathrm{~mL}$ in the HPVP group, which was remarkably high. This may be related to the surgical technique used in this study, as the authors routinely perform a spinal tap to break the wall of the cavity composed by necrotic and sclerotic bones. Yu et al. ${ }^{18}$ demonstrated that long-term radiologic and clinical outcomes of a diffused bone cement pattern were superior to those of a solid lump distribution pattern for osteoporotic vertebral compression fractures with an intravertebral cleft. If the wall was not broken, a diffused bone cement pattern was hard to obtain. Moreover, a previous study reported that KD was a strong protective factor for cement leakage, ${ }^{19}$ which may be explained by the layer of dense sclerotic bone around the cavity of the vertebral body, preventing bone cement leakage. However, in the present work, the approach at breaking the sclerotic belt may be the main reason the cement leakage rate was significantly higher than that of the other studies. Regarding the mechanism as to why the cement leakage rate was higher in the NPVP group than in the HPVP group, the authors thought that the cavity might be small in the neutral position, and the upper endplate and sclerotic belt are closer to the lower endplate and sclerotic belt. Thus, either the upper or lower endplate can break easily during the spinal tap. However, in the hyperextension position, the cavity is large, and the upper endplate and sclerotic belt are farther from the lower endplate and sclerotic belt; hence, breaking it by spinal tap is difficult.

This study has some limitations. First, this was a retrospective comparative study, and no method was used to ensure the unbiased randomisation of the two groups. Additionally, the patient sample size was small, and data on longer follow-up clinical outcomes were unclear due to the finite follow-up duration. A prospective randomised controlled study with a longer follow-up period would better assess the clinical outcomes of HPVP for treating KD.

\section{CONCLUSION}

The results of this study suggest that HPVP can achieve better VAS, ODI, Cobb's angle, and AR at the one-year follow-up than NPVP, without prolonging the operative time. Additionally, the cement leakage rate is lower with HPVP. Thus, HPVP was superior to NPVP in treating KD, and the authors recommend it as the preferable position for this kind of surgery.

\section{DISCLOSURE:}

The authors declared that they are not part of any thesis or dissertation, a pilot project, or an ongoing study.

\section{FUNDING:}

This study was supported by Yantai Science and Technology Plan (2020YD039).

\section{ACKNOWLEDGEMENT:}

The authors would like to thank Editage (www.editage.com) for English language and publication support.

\section{ETHICAL APPROVAL:}

This study was conducted in accordance with the World Medical Association Declaration of Helsinki and was approved by the Ethics Committee of Yantaishan Hospital (approval No. 2020002).

\section{PATIENTS' CONSENT:}

The written consents for the publication of the images included in this study were obtained from all the patients and their relatives.

\section{CONFLICT OF INTEREST:}

All authors declared no conflict of interest.

\section{AUTHORS' CONTRIBUTION:}

YD: Contributed to the conception of the work, draft writing.

SD: Contributed to data analysis and interpretation, draft revision.

JW, JC, ZC: Contributed to acquisition of data and analysis

SL: Contributed to conception of the study, critical revision, final approval of the version to be published and agreement to be accountable for all aspects of the work. 


\section{REFERENCES}

1. Ma R, Chow R, Shen FH. Kummell's disease: Delayed posttraumatic osteonecrosis of the vertebral body. Eur Spine J 2010; 19(7):1065-70. doi.org/10.1007/ s00586-009-1205-4.

2. Huang $Y$, Peng $M$, He S, Tang $X$, Dai M, Tang C. Clinical efficacy of percutaneous kyphoplasty at the hyperex-tension position for the treatment of osteoporotic Kümmell disease. Clin Spine Surg 2016; 29(4):161-6. doi.org/ 10.1097/BSD.0000000000000259.

3. Zhang J, Fan $Y$, He X, Meng $Y$, Huang $Y$, Jia S, et al. Is percutaneous kyphoplasty the better choice for minimally invasive treatment of neurologically intact osteoporotic Kümmell's disease? A comparison of two minimally invasive procedures. Int Orthop 2018; 42(6):1321-6. doi.org/10. 1007/s00264-018-3832-z

4. Chen GD, Lu Q, Wang GL, Zou J, Yang HL, Yang Y, et al. Percutaneous kyphoplasty for Kummell disease with severe spinal canal stenosis. Pain Physician 2015; 18:E1021-8.

5. Zhang Y, Yang H, Liu Y, Zhou F, Deng X, Luo W, et al. Percutaneous kyphoplasty in hyperextension position for treatment of middle and late period Kümmell disease. Zhongguo Xiu Fu Chong Jian Wai Ke Za Zhi 2012; 26:411-5.

6. Kim P, Kim SW. Balloon kyphoplasty: An effective treatment for Kummell disease? Korean J Spine 2016; 13(3):102-6. http://doi.org/10.14245/kjs.2016.13.3.102

7. Qi Y, Zeng $Y$, Jiang C, Liang B, Sui J, Zhao L, et al. Comparison of percutaneous kyphoplasty versus modified percutaneous kyphoplasty for treatment of osteoporotic vertebral compression fractures. World Neurosurg 2019; 122:e1020-7. doi.org/10.1016/j.wneu.2018.10.205.

8. Park JW, Park JH, Jeon HJ, Lee JY, Cho BM, Park SH. Kümmell's disease treated with percutaneous vertebroplasty: minimum 1 year follow-up. Korean J Neurotrauma 2017; 13(2):119-23. doi.org/10.13004/ kjnt.2017.13.2.119.

9. He D, Yu W, Chen Z, Li L, Zhu K, Fan S. Pathogenesis of the intravertebral vacuum of Kümmell's disease. Exp Ther Med 2016; 12(2):879-82. doi.org/10.3892/etm.2016.3369

10. Yang S, Chen C, Wang H, Wu Z, Liu L. A systematic review of unilateral versus bilateral percutaneous vertebroplasty/percutaneous kyphoplasty for osteoporotic vertebral compression fractures. Acta Orthop Traumatol Turc 2017; 51:290-7. doi.org/10.1016/j.aott.2017.05.006.

11. Chen W, Xie W, Xiao Z, Chen H, Jin D, Ding J. Incidence of cement leakage between unilateral and bilateral percutaneous vertebral augmentation for osteoporotic vertebral compression fractures: a meta-analysis of randomized controlled trials. World Neurosurg 2019; 122:342-8. doi.org/10.1016/j.wneu.2018.10.143.

12. Wang H, Hu P, Wu D, Zhang N, Wu J, Xiang L. Age, gender, level and side differences in the anatomical distinctions of unilateral percutaneous kyphoplasty through the transverse process-pedicle approach. Pain Physician 2019; 22(2): E91-6.

13. Laredo JD. Expert's comment concerning Grand Rounds case entitled "Kümmell's disease: delayed post-traumatic osteonecrosis of the vertebral body" (by R. Ma, R. Chow, F. H. Shen). Eur Spine J 2010; 19:1071-2. doi.org/10.1007/ s00586-009-1204-5.

14. Ryan PJ, Blake G, Herd R, Fogelman. A clinical profile of back pain and disability in patients with spinal osteoporosis. Bone 1994; 15(1):27-30. doi.org/10.1016/ 8756-3282 (94)90887-7.

15. Minamide A, Maeda T, Yamada H, Murakami K, Okada M, Enyo $Y$, et al. Early versus delayed kyphoplasty for thoracolumbar osteoporotic vertebral fractures: The effect of timing on clinical and radiographic outcomes and subsequent compression fractures. Clin Neurol Neurosurg 2018; 173:176-81. doi.org/10.1016/j.clineuro.2018.07.019.

16. Lindsay R, Silverman SL, Cooper C, Hanley DA, Barton I, Broy $\mathrm{SB}$, et al. Risk of new vertebral fracture in the year following a fracture. JAMA 2001; 285(3):320-3. doi.org/ 10.1001/jama.285.3.320.

17. Ng JP, Cawley DT, Beecher SM, Baker JF, McCabe JP. The reverse thomas position for thoracolumbar fracture height restoration: Relative contribution of patient positioning in percutaneous balloon kyphoplasty for acute vertebral compressions. Int J Spine Surg 2016; 10:21. doi.org/10. $14444 / 3021$.

18. Yu W, Xiao X, Zhang J, Li Z, Wang X, Tang F, et al. Cement distribution patterns in osteoporotic vertebral compression fractures with intravertebral cleft: effect on therapeutic efficacy. World Neurosurg 2019; 123:e408-15. doi.org/ 10.1016/j. wneu.2018.11.181.

19. Tomé-Bermejo F, Piñera AR, Duran-Álvarez C, Lopez-San Roman B, Mahillo I, Alvarez $\mathrm{L}$, et al. Identification of risk factors for the occurrence of cement leakage during percutaneous vertebroplasty for painful osteoporotic or malignant vertebral fracture. Spine 2014; $39(11)$ : E693-700. doi.org/10.1097/brs.0000000000000294. 